\title{
Jugendbewegungen im Netz
}

L im Landkreis Nordsachsen

\author{
Digitale Kommunikationsstrategien der „Identitären Bewegung”
}

\author{
von Prof. Dr. Falk Tennert
}

Die „Identitäre Bewegung“ (IB) als eine Strömung der hiesigen und international Neuen Rechten versteht sich als europaweite, aktionistisch orientierte Jugendbewegung. Ihr priorisiertes Themenfeld bezieht sich auf die Gefährdung der kulturellen Identität Europas durch die zunehmende „Islamisierung“, die Ablehnung der multikulturellen Gesellschaft und Widerstand gegen außereuropäische Einwanderung (Camus 2017: 233). Vor dem Hintergrund dieser von der IB erkannten Entwicklungen sieht sie sich als eine neue politische Protestbewegung, deren Betätigungsfeld das Bewahren nationaler Identitäten und Kulturräume ist („Nicht links, nicht rechts - identitär!“) und setzt damit auf das Konzept des so genannten Ethnopluralismus. Hiernach wird eine kulturelle „Reinhaltung“ von Staaten oder Gesellschaften angestrebt, was - wissenschaftlich unhaltbar - von Primordialität ausgeht und in der Umsetzung klar rassistisch ist. Die IB nutzt in ihrer weitgehend digitalen Protest- und Kommunikationsstrategie erkennbare Codes (wie das schwarz-gelbe Lambda) und ein popkulturelles Erscheinungsbild. Hierbei werden Elemente der Guerilla-Kommunikation verwendet, so dass die Aktionen überraschend, idealerweise spektakulär und rebellisch erscheinen. Bilder und Videos dieser Protestaktionen sowie Interviews mit den Aktivisten der IB werden in sozialen Medien wie Facebook oder auf Videoplattformen wie Youtube bereitgestellt. Neben den Protestaktionen werden auch scheinbar unpolitische Formate genutzt: „IBster Küche“ heißt beispielsweise eine Kochshow auf Youtube mit Martin Sellner, einem der führenden Köpfe der IB, in der er ein (nicht ganz echtes) Wiener Schnitzel zubereitet und den Herstellungsprozess gesellschaftspolitisch framt. Niedrigschwellige Angebote wie Kochshows, Interviews und Comedy-Formate rechter Influencer oder Games dienen als Lockmittel, in denen die Buzzwords identitärer Themen gesetzt werden, eingebettet in tradierte Codes popkultureller Milieus.

Der Beitrag diskutiert zunächst Merkmale des sozial-psychologischen Konstrukts Identität. Im Anschluss daran werden die Öffentlichkeitsstrategien der „Identitären Bewegung“ in Deutschland dargestellt, um die Strukturen IB-bezogener Themensetzung im Rahmen der (präsenz-) öffentlichen wie digitalen Kommunikation herauszuarbeiten.

\section{Die „Identitäre Bewegung“ in Deutschland}

2014 gründete sich die „Identitäre Bewegung“ in Deutschland (IBD). Die Idee stammt aus Frankreich, wo der „Bloc Identitaire“ seit 2003 mit islamkritischen Botschaften vor allem in den sozialen Medien aktiv ist. „Die Werte Heimat, Freiheit und Tradition spielen im Selbstverständnis der IBD als Teil einer europaweiten patriotischen Jugendbewegung eine große Rolle. Die IBD verfolgt das Ziel, mit gemeinschaftlichen Aktivitäten und kulturellen sowie politischen Bildungsangeboten für diese Werte einzutreten. Die IBD bekennt sich zum Konzept des Ethnopluralismus, nach dem die Idealvorstellung einer staatlichen beziehungsweise gesellschaftlichen Ordnung in einem ethnisch und kulturell homogenen Staat besteht.“ (BfV, Verfassungsschutzbericht 2018: 82). Diese ethnokulturelle Identität sieht die IB durch den sogenannten Multikulturalismus bedroht, der durch eine vor allem muslimisch geprägte Zuwanderung zu einer Heterogenisierung der Gesellschaft führe. Daher

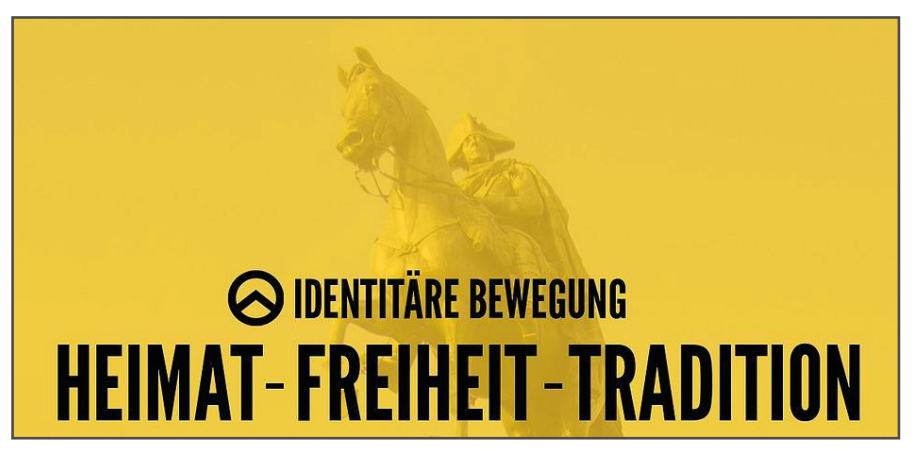

fordert sie im Rahmen ihrer Kampagnen unter dem Schlagwort „Remigration" Maßnahmen zur Umkehrung der Flüchtlingsströme und die Rückführung von Migranten in deren Heimatländer. Kritisiert wird auch die Asylpolitik vieler europäischer Staaten als Förderung des „Großen Austauschs“, wonach die Mehrheitsgesellschaft gegen Migranten ausgetauscht werde und warnt vor einer „Islamisierung“ Deutschlands. Die „Identitäre Bewegung" bezieht sich somit maßgeblich auf ethnische, also völkisch-abstammungsorientierte Kriterien. Vor allem die Fokussierung auf eine ethnische Homogenität als zentralem Gesellschaftswert stellt einen deutlichen Anhaltspunkt dafür dar, ,dass die Ideologie der IBD die grundgesetzlich geschützte Menschenwürde und das Demokratieprinzip verletzen könnte.“ (BfV, Verfassungsschutzbericht 2018: 83); daher beobachtet das BfV die IBD als Verdachtsfall. Die IB vertritt insofern einen völkischen Staatsvolk-Begriff, der dem Verständnis des Grundgesetzes gemäß Art. 116 GG widerspricht. Unter anderem definiert sie „Staatsvolk als Kultur-, Abstammungs- und Solidargemeinschaft", wobei sie der Ethnie als der maßgeblichen Grundlage für Kultur und gesellschaftlichen Zusammenhalt stets den Vorrang einräumt.

\section{„Identitäre Bewusstsein"}

Für die IB ist der Nationalstaat der zentrale Vermittlungsrahmen zwischen der Verwurzelung in einer Region - im Sinne des Begriffs Heimat - und der Zugehörigkeit in einem übergeordneten, europäischen Zivilisationszusammenhang. Was bedeutet nun eigentlich Identität? Sozialpsychologisch betrachtet handelt es sich bei Identität um die Fragestellung nach dem Selbst. Die Herausbildung des Selbst resultiert aus einer wechselseitigen Beziehung zwischen Eigen- und Fremdwahrnehmung. Diese Wechselseitigkeit basiert u.a. auf gemeinsam geteilten Lebenswelten sowie Interaktions- und Kommunikationsprozessen. Neben einer individuellen Identität existieren auch gruppenbezogene, soziale Identitäten. Solche gruppenbezogenen Identitäten können sich als Fan-Verhalten zeigen, als Nähe zu politischen Parteien, zu Marken oder Nationen. Die gruppenbezogene Identität ist somit jener Teil des Selbstkonzeptes einer Person, der sich aus dem Wissen über die Mitgliedschaft in oder Nähe zu einer sozialen Gruppe ableitet, einschließlich des Wertes und der emotionalen Bedeutung, die daraus resultiert
ISSN 2629-5784 Transfer Plus - Akłuelle Beiträge zur Medienbildung

In Transfer ${ }^{\text {Plus }}$ besprechen Autoren aus Wissenschaft und Forschung leicht verständlich und prägnant aktuelle Befunde aus Journalismus, Medien und Gesellschaft. Literatur- und Medientipps geben Anregungen $\mathrm{z}$ Vertiefung und Thematisierung in der Bildungsarbeit. TransferPlus wird auf Qucosa, dem sächsischen Open-

Herausgeber

Redaktion 
(Simon \& Trötschel 2007: 171 f.). Der Grundgedanke hierbei ist der, dass die Zugehörigkeit zu einer bestimmten Gruppe das Selbst definiert und formt: „Je nach Gruppenzugehörigkeit verfügen Menschen nun über mehrere Selbstdefinitionen, die einen Teil ihres Selbstkonzepts determinieren. Jede Mitgliedschaft stellt eine kognitive Repräsentation (soziale Identität) dar, die das Individuum zugleich beschreibt und auch in seinen individuellen Kognitionen, Aktionen und Emotionen festlegt.“ (Müller 2011: 53). Jede gruppenbezogene Identität wird dabei je nach Situation verschieden erlebt und besitzt eine unterschiedliche individuelle Prägekraft: so in der Rolle als Fan einer Fußballmannschaft, als Familienvater, als Mitglied einer Partei, als Deutscher oder als Europäer. Welche Identität vorrangig empfunden und kultiviert wird, hängt vom Lebensalter, dem Kontext und der jeweiligen Lebenssituation ab. Die unterschiedlichen sozialen Identitäten stehen dabei in einem wechselseitigen, situativen Verhältnis, wobei Menschen üblicherweise über eine Hauptidentität verfügen, die eine implizierte Organisationsstruktur aller anderen Identitäten beinhaltet.

Welche Funktionen erfüllt die gruppenbezogene Identität für eine Person? Diese Form von Identität schafft eine Verfestigung von Ich-Eigenschaften in Bezug auf eine Gruppe. Wofür stehe ich? Welche Werte teilt die Gruppe? Dabei stehen Kontinuität und Realitätssicherung im Sinne von gemeinsamen Einstellungen, Erfahrungen, Werten, Handlungsweisen und Zukunftserwartungen durch gruppenbezogene Identitäten im Fokus. Die Identitätsherausbildung entsteht dabei durch eine Wechselbeziehung zwischen Individuum und sozialer Umwelt als kontinuierlichem Differenzierungs- und Wandlungsprozess. Insofern lassen sich Komponenten regionaler, nationaler und europäischer Identitäten grundsätzlich miteinander verbinden, je nach situativer und individueller Relevanz. Eigene Erfahrungen werden so durch soziale Handlungen und Wissensbestände gedeutet und tragen damit zur Verstärkung des Wir-Gefühls bei (Schlee 2017: 213 ff.).

Der sozialen Identitätskonstruktion kommen dabei verschiedene Funktionen zu: Erstens erleichtert sie die Einordnung von aktuellen Erfahrungen, zweitens reduziert sie Komplexität und ermöglicht damit eine Orientierung in der Gesellschaft, und drittens ist Identität das Resultat vergangener Identifizierungsprozesse. Das Wir-Gefühl schafft Nähe zur eigenen Gruppe und Abgrenzung zu anderen sozialen Gruppen. Erst diese Distinktion ermöglicht es überhaupt, Differenzen zu anderen erlebbar und beschreibbar zu machen. Für die Eigengruppe nimmt man sich als ähnliches Mitglied wahr und gleichzeitig als unterscheidbar zu Mitgliedern der Fremdgruppe. Im Zuge der Abgrenzung und Identitätsfindung spielen gruppenkonstituierende Attribute, also Merkmale, wofür eine Gruppe steht, eine besondere Bedeutung. Das Herausheben spezifischer Attribute oder Codes (bspw. Zeichen, Kleidung, gemeinsame Werte) ist hierbei zentral; dies bezeichnet man als Salienz der sozialen Identität. Salienz fördert die innere Homogenisierung der Gruppe, die Einebnung von Realitätswahrnehmungen und eine spezifische Selbstwertschätzung über das Wir-Gefühl. Je nach Art der Erfahrung und Deutung prägt das gruppenbezogene Selbst Alltagswahrnehmungen, Deutungen der Gesellschaft und Vorstellungen von einer Zukunft.

Interaktions- und Kommunikationsprozesse stellen zentrale Bedingungen für die Entstehung gruppenbezogener Identitäten dar. Dies geschieht beispielsweise durch Symbole, Farben oder Kleidung als äußere und sichtbare Begrenzungen von Gemeinschaften. Codes als Identitätsmarker reduzieren im sozialen Miteinander Komplexität und schaffen so eine schnelle Wiedererkennbarkeit und Abgrenzung zu anderen Gruppen. Zur Kategorisierung sozialer Identitäten lassen sich drei verschiedene Code-Ebenen heranziehen (Giessen 1999: 130 ff.):

- Der primordiale Code bezieht sich auf die „Naturalisierung“ von Identität und umfasst Merkmale wie Geschlecht, Ethnie oder die Bindung an ein bestimmtes Territorium.

- Der konventionelle Code bezieht sich auf kollektive Verhaltensregeln und soziale Routinen, etwa der Praxis des Gemeinwesens, der Staatsbürgerlichkeit oder etablierter Umgangsformen.

- Und beim kulturellen Code handelt es sich um konkret sichtbare Zeichen: Mit Hilfe von Emblemen, mit denen man sich zur kulturellen Gemeinschaft bekennt, wie Aufkleber und Kleidungsstücke, Haartracht und Accessoires, aber auch durch Texte und Lieder werden kulturelle Codes angezeigt. Bei der „Identitären Bewegung“ werden solche Identitätsmarker beispielsweise durch die Vermarktung von T-Shirts, Hoodies und Aufklebern oder durch Fahnen mit dem Lambda-Symbol bereitgestellt (vgl. www.ibladen.de).

\section{Warum erstellt die IB Kochvideos?}

Die Konstruktion von Gesellschaftsbildern im Sinne einer gruppenbezogenen Identität vollzieht sich bei der IB vor allem im (präsenz-)öffentlichen und digitalen Raum. Live vor Ort aufgenommene Fotos und Videos von Aktionen werden in den sozialen Netzwerken bereitgestellt und geteilt, um so eine junge, digital affine Generation anzusprechen. Neben der Information über die Kernthemen der IB zielen die Angebote vor allem auf soziale Funktionen von Kommunikation wie Integration (Herstellen eines Gemeinschaftsgefühls in einer fragmentierten Welt,

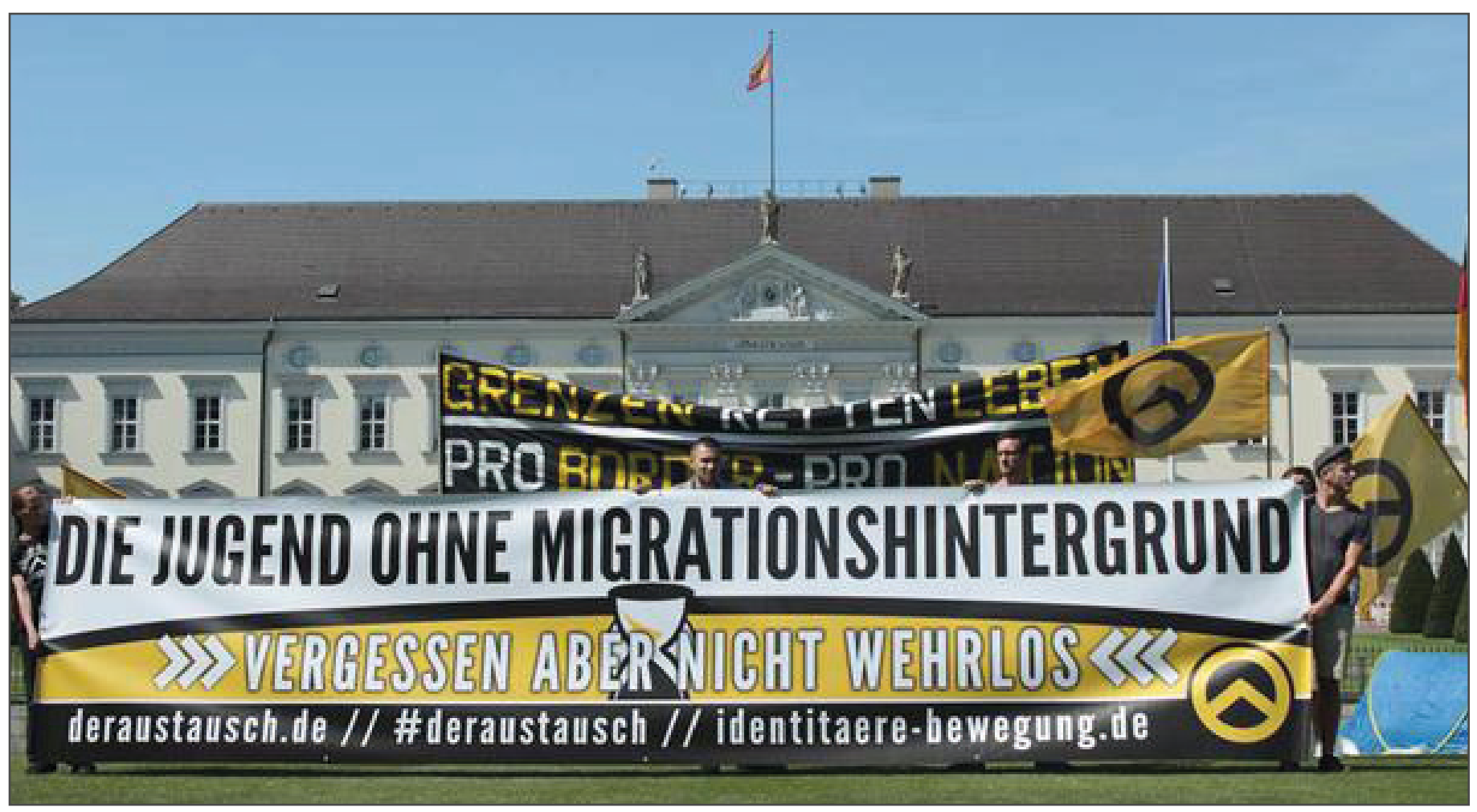




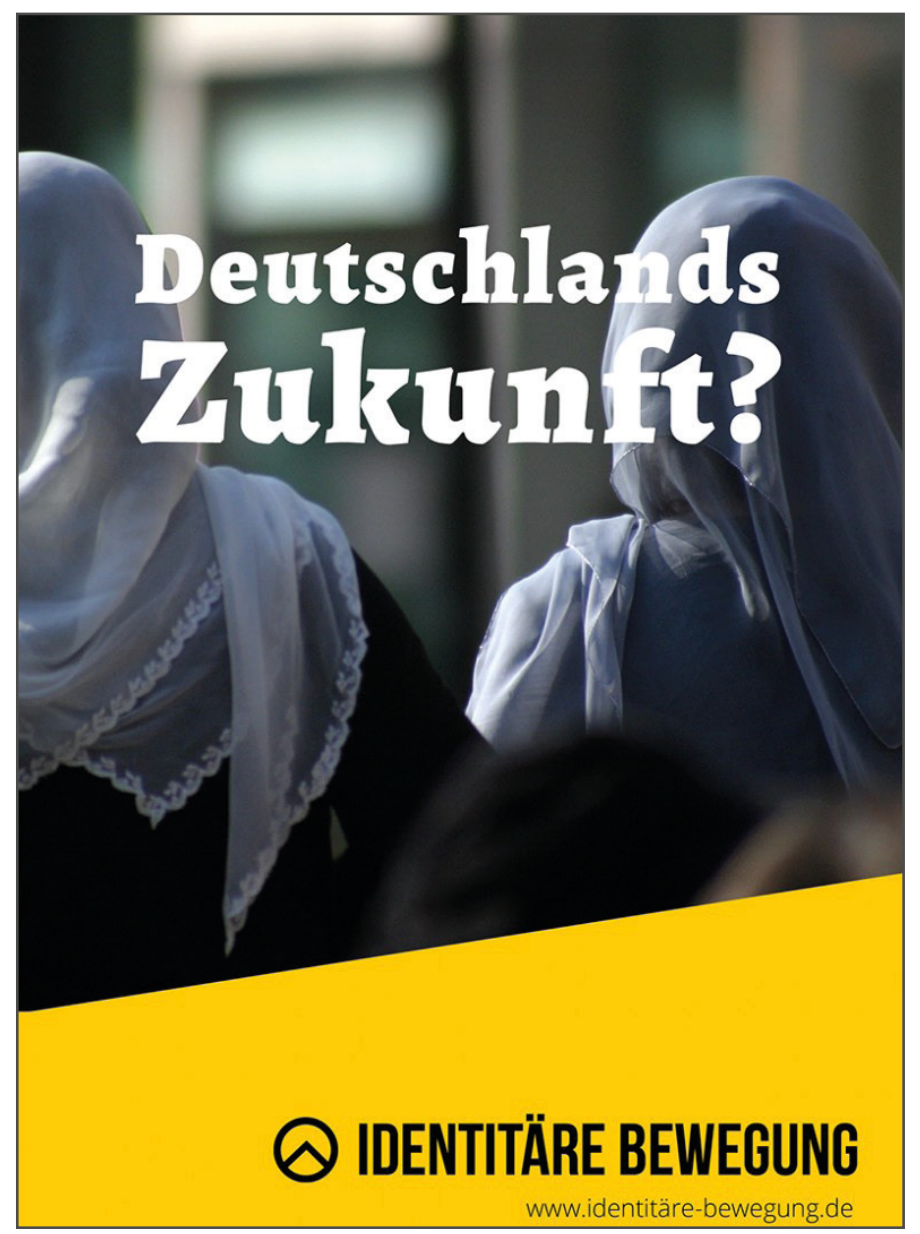

Protestthemen als Basis für Anschlusskommunikation), soziale Orientierung und auch Unterhaltung (Beck 2017: $101 \mathrm{ff}$.), bspw. durch Videos und Games. Digitale Publizität wird über die Nutzung von Videoplattformen, Social Communities und Microblogs sowie entsprechender Hashtags \#defendeurope oder \#deraustausch hergestellt. Zentraler Ausgangspunkt sind hier die Kernthemen außereuropäische Migrationsbewegungen und Multikulturalismus in Europa, aber auch soziale Fragen vor dem Hintergrund der Globalisierung oder eine wahrgenommene eingeschränkte Meinungspluralität in den Medien („Okzident News Die patriotische Nachrichten-App“). Die Reichweiten dieser digitalen Formate sind im Vergleich zu anderen Influencern allerdings marginal, die Verfügbarkeit von Apps oder Profilen ist häufig von kurzzeitiger Dauer. Der bekannteste deutschsprachige Influencer der „Identitären Bewegung" hat auf Youtube 120.000 Abonnementen, IBster Küche wurde 39.000 Mal angesehen. Auch eine durch Crowdfunding 2017 finanzierte App namens „Patriot Peer" funktioniert nicht. Formen, Inhalte und Reichweite dieser Kanäle sind weitgehend ausgereizt. Neben digitalen Kanälen wird auf präsenzöffentliche Protestaktionen gesetzt. Hierzu einige Beispiele: Die IBD trat im Jahr 2018 mit dem „Europa Nostra"-Festival in Dresden in Erscheinung; das Programm umfasste neben Reden und Vorstellungen verschiedener Organisationen auch Verkaufs- und Ausstellungsstände. Auch die 2018 initiierte Kampagne "Migrationspakt stoppen“ führte zu verschiedenen Flashmob-Aktion in Berlin und Bautzen („UN-MIGRATIONSPAKT STOPPEN“). Mit „Mission Alpes“ fand die Kampagne „Defend Europe“ im Frühjahr 2018 ihre Fortsetzung. Im französisch-italienischen Grenzgebiet errichtete eine europäisch zusammengesetzte Gruppe von Aktivisten der IB eine symbolische Grenze, um Migranten an der Reise nach Mitteleuropa zu hindern. Im August 2016 kletterten Vertreter der IBD auf das Brandenburger Tor und entrollten Großbanner, auf denen „Sichere Grenzen - sichere Zukunft“ und „Grenzen schützen - Leben retten“" standen (zusammenfassend BfV 2018, S. 83 f.). Diese aktuellen Aktionen waren im Vergleich zur Frühphase der IBD wenig erfolgreich. Mit der Durchführung solcher ursprünglich im politisch linken Milieu entstandenen „subversiven Aktionen“ schafft die IB inneren Zusammenhalt und (limitierte) öffentliche Aufmerksamkeit. Die Aktionen an sich stehen als Träger der Botschaften im Vordergrund. Die präsenzöffentliche Reichweite ist gering, die Kampagnen sind vorrangig für die Netzöffentlichkeit angelegt. Die aus den Aktionen resultierende Medienreso-nanz in Tages- und Wochenzeitungen oder Fernsehsendungen ist Teil der Kommunikationsstrategie. Zentrale Merkmale der digitalen Netzöffentlichkeit sind u.a. die Umkehrung der klassischen (massenmedialen) Sender-Empfänger-Beziehung und damit die Umgehung journalistischer Gatekeeper, um eine themenzentrierte „Gegenöffentlichkeit" zu erzielen.

Betätigungsfelder der „Identitären Bewegung“ sind vor allem der Bildungsbereich (Hochschulen), Medieneinrichtungen (v.a. Sendeanstalten des öffentlich-rechtlichen Rundfunks), Parteizentralen (u.a. SPD-Zentrale in Berlin) oder Kultureinrichtungen. Dabei nutzt die IB etablierte Stilelemente im Sinne einer Corporate Identity, um somit eine eindeutige Zuordnung oder Wiedererkennung zu ermöglichen. Weiterhin werden für die präsenzöffentliche wie digitale Kommunikationsstrategie Ästhetiken genutzt, die provokativ wie anschlussfähig gleichermaßen sind. Dies erfolgt durch eine Referenz auf kommunikative Merkmale, die im kollektiven Gedächtnis eher zivilgesellschaftlichen Aktions- oder Protestformen zuzuordnen sind, so etwa stilistische Bezüge zum studentischen, kreativen oder großstädtischen Milieu: ,, Man kleidet sich modisch und sportlich, man vermeidet martialisches Auftreten im Alltag und setzt zugleich auf die subkulturell inspirierte Ästhetisierung der eigenen politischen Botschaft - zur Abgrenzung gegen den äußeren Rest der politischen Welt und zur sichtbaren identitären Gemeinschaftsbildung nach innen. " (Boehnke 2019: 90).

Neben diesen protestbezogenen Formaten werden auch unterhaltungsorientierte Angebote erstellt (IBster Küche auf Youtube) oder Produkte der IB vermarktet (bspw. das Bier Pils identitär: natürlich - patriotisch - lokal). Durch die oben genannten Thematisierungsstrategien werden „Rahmenelemente“ zur Identitätskonstruktion hergestellt. Der Begriff des Rahmens (Framing) verdeutlicht, dass es um die Schaffung eines Deutungsraums geht, der so konstruiert ist, dass die Perspektive auf Aspekte jenseits des Deutungsraumes eingeschränkt wird und innerhalb dessen bestimmte Merkmale moralisch aufgewertet werden. Durch ein solches Framing wird der Fokus auf spezifische Themenaspekte, Ideen oder Ideologien hergestellt, interpretiert und zu Zusammenhängen geformt, um bestimmte politische Ziele zu verwirklichen. Dies geschieht bspw. durch die einseitige Gewichtung auf negative Aspekte von Migration oder - bei scheinbar unpolitischen Formaten - die IBster Küche als ,praktizierter Ethnopluralismus“. Mit dieser bewussten Rahmung durch Aktivisten der IB wird politische Identität geschaffen, indem Ereignisse, Erfahrungen und Ideologiestränge zu einer kollektiv und prozesshaft konstruierten Bedeutungseinheit verknüpft werden.

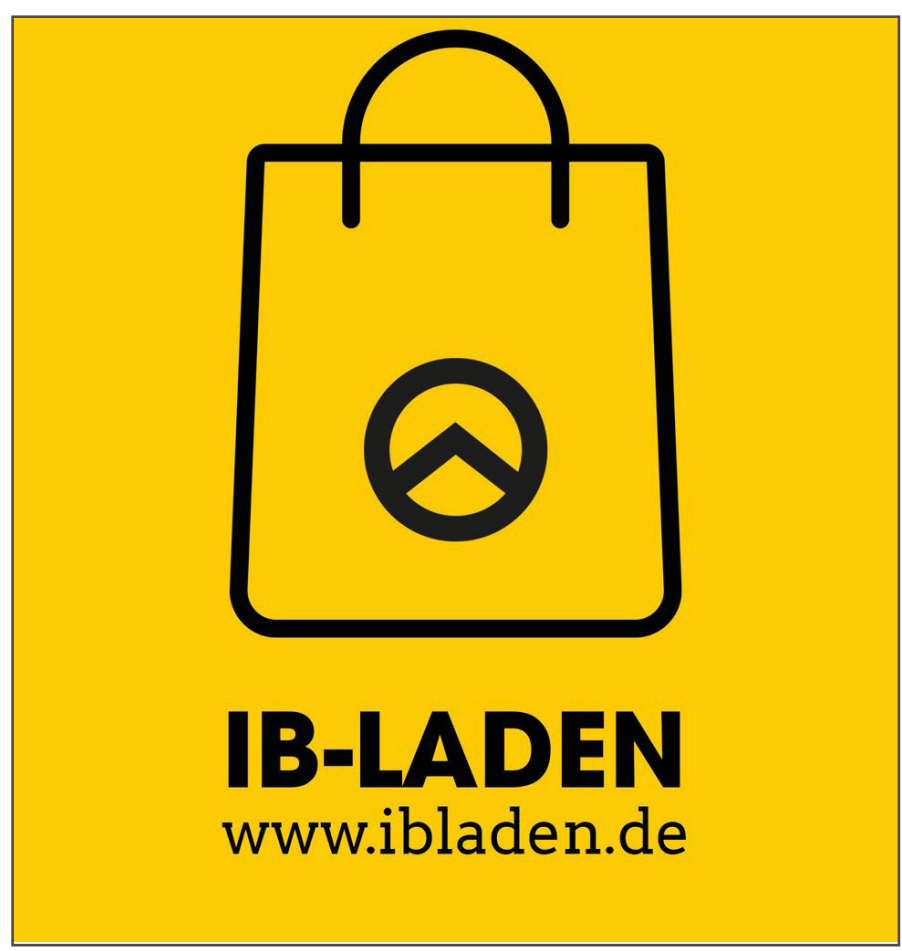




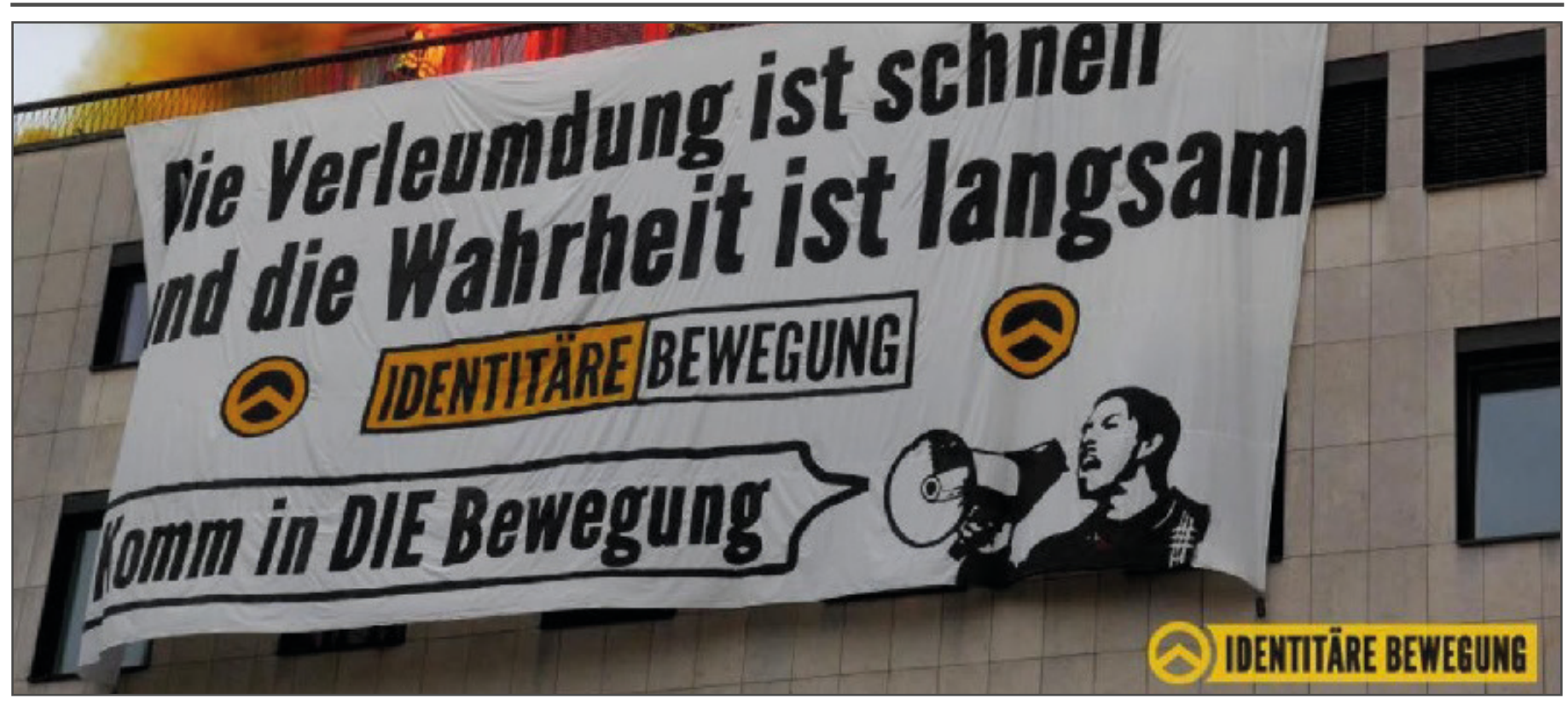

\section{Schlussfolgerungen}

Die neurechte „Identitäre Bewegung“ war zunächst als rein virtuelle Erscheinung aktiv und hat durch verschiedene Aktionsformen, Kampagnen und Festivals den Sprung in die reale Welt vollzogen. Die Werte „Heimat, Freiheit und Tradition" spielen im gruppenbezogenen Selbstverständnis der IB als Teil einer europaweiten patriotischen Jugendbewegung eine zentrale Rolle (BfV 2018). Als eine neurechte Strömung hat es die IB verstanden, digitale Medien für sich zu nutzen und durch subversive Formate öffentliche Aufmerksamkeit zu generieren. Die Aktionsformen sind stark an kommunikative Guerilla-Strategien angelehnt, als innovative und unkonventionelle Kommunikationsformate, die gleichzeitig umfangreiche popkulturelle Inhalte samplen, sich mit zunehmendem Einsatz jedoch auch schnell verbrauchen.

\section{Handlungsempfehlungen}

Folgende medienpädagogische Handlungsempfehlungen können gegeben werden: (i) Identitäre Codes und Themen erkennen. Hier geht es darum, Themen und Codes der „Identitären Bewegung“ zu erkennen (Corporate Identity, Ästhetik, Codes). Fotos, Videos oder Aufkleber der IB tragen nicht immer deren Namen, sondern mitunter gibt nur das Lambda einen Hinweis. Das gilt nicht nur für die „Straße“, sondern auch für digitale Medien, in denen die Inhalte schnell geteilt werden ohne dass der Hintergrund bekannt ist. (ii) Diskursive Auseinandersetzung mit Beiträgen der IB. Die Themen der IB greifen gesellschaftliche Konfliktlinien wie Migrationsbewegungen in Europa auf. Migration wird aus Sicht der IB nicht nur als Kulturproblem, sondern auch als Herausforderung für den Sozialstaat aufgefasst. In dieser Hinsicht kann eine diskursive Auseinandersetzung mit Positionen der Neuen Rechten erfolgen, eine solche komparative Auseinandersetzung kann auch Querverbindungen zu verwandten Konzepten der AfD oder zu Pegida umfassen. (iii) Deutungsrahmen analysieren: Als soziale Bewegung nutzt die IB verschiedene Elemente der Partizipation, um so auf die öffentliche Themensetzung Einfluss zu nehmen. Durch Framing und Agenda Building wird ein strategischer Deutungsrahmen für die öffentliche Kommunikation vor allem in den sozialen Medien generiert. Dies erfolgt durch eine Referenz auf kommunikative Merkmale, die stilistische Bezüge zum studentischen oder großstädtischen Milieu aufweisen.

\section{Quellen}

Beck, K. (2017): Kommunikationswissenschaft. Konstanz: UVK

Böhnke, L. (2019): Rechter Kulturkampf heute: Identitätskonstruktion und FramingStrategien der Identitären Bewegung. In: Böhnke, L.; Thran, M. \& J. Wunderwald (Hrsg.): Rechtspopulismus im Fokus. Wiesbaden: Springer VS, S. 89-114.

Bundesministerium des Innern, für Bau und Heimat (Hrsg.): Verfassungsschutzbericht 2018. Berlin.

Camus, J.-Y. (2017): Die Identitäre Bewegung oder die Konstruktion eines Mythos europäischer Ursprünge. In: Hentges, G.; Nottbohm, K. \& Platzer, H.-W. (Hrsg.) Europäische Identität in der Krise? Wiesbaden: Springer VS, S. 233-248.

Giesen, B. (1999): Europa als Konstruktion der Intellektuellen. In: Viehoff, R.; Segers, R.T., (Hrsg.): Kultur, Identität, Europa. Frankfurt (Main): Suhrkamp, S. 130-146. Müller, B. (2011): Empirische Identitätsforschung: Personale, soziale und kulturelle Dimensionen der Selbstverortung. Wiesbaden: Springer VS.

Schlee, G. (2017) Interethnische Beziehungen. In: Beer, B.; Fischer, H. \& Pauli, J. (Hrsg.): Ethnologie: Einführung in die Erforschung kultureller Vielfalt. Berlin: Reimer, S. 213228.

Simon, B.; Trötschel, R. (2007): Das Selbst und die soziale Identität. In: Jonas, K. Stroebe, W. \& Hewstone, M. (Hrsg.): Sozialpsychologie. Wiesbaden: Springer VS, S. $147-185$

Autor der Ausgabe

Falk Tennert ist Professor für Empirische Forschungsmethoden an der Berufsakademie Sachsen, Staatliche Studienakademie Breitenbrunn. Kontakt: f.tennert@ba-breitenbrunn.de

\section{Empiohlene Zifation des Beitrages}

Tennert, F. (2020). Jugendbewegungen im Netz. Digitale Kommunikationsstrategien der „Identitären Bewegung”. In: B. Bigl (Hrsg.). Transfer - Beiträge zur Medienbildung. Issue 01/2020. doi:10.25366/2020.12

Links

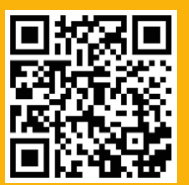

Neue Rechte auf dem Vormarsch (Panorama 3, NDR)

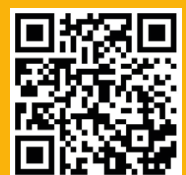

Demonstration den IB in Berlin (SPIEGEL Online)

Literatur

Abels, H. (2017): Identität. Wiesbaden: Springer.

Bötticher, A.; Mareš, M. (2012): Extremismus: Theorien, Konzepte, Formen. München: Oldenbourg Wissenschaftsverlag.

Hentges, G.; Nottbohm, K. \& Platzer, H.-W. (2017): Europäische Identität in der Krise? Europäische Identitätsforschung und

Rechtspopulismusforschung im Dialog. Wiesbaden: Springer. 